\title{
Registries, Diagnostic Studies, and Therapeutic Interventional Trials: Generating Evidence in a Broad Perspective
}

\author{
Register, diagnostische und Therapiestudien: Ihre Beiträge zur evidenzbasierten Medizin
}

\section{T. Schneider}

Affiliation

Clinic of Pediatrics, Dortmund Municipal, Dortmund, Germany

\section{Bibliography}

DOI http://dx.doi.org/ 10.1055/s-0033-1357173

Klin Padiatr 2013; 225: 293-295

(C) Georg Thieme Verlag KG

Stuttgart · New York

ISSN 0300-8630

\section{Correspondence}

Prof. Dr. Dominik T. Schneider

Clinic of Pediatrics

Dortmund Municipal Hospital

Beurhausstraße 40

44137 Dortmund

Tel.: + 49/231/953 21680

Fax: +49/231/953 21047

dominik.schneider@klini

kumdo.de
Evidence-based medicine is one of the most prominent keywords in modern medicine. According to the concepts of evidence-based medicine, different levels of evidence are defined. These may range from expert opinion or single case reports to clinical studies, prospective randomized trials and ultimately the meta-analysis of several prospective randomized controlled trials (RCT). In interdisciplinary consensus guidelines, the different levels of evidence are translated into recommendations at different strengths $[5,7,17]$. As these guidelines reflect the current standard of care, they serve as an important orientation in the clinical management of patients. Furthermore, they facilitate harmonization of treatment, in particular in an interdisciplinary or international setting $[10,11]$.

Different types of studies can be distinguished, among others interventional clinical trials, diagnostic, prognostic, and quality of life studies. In all, hypotheses are tested that have been developed prospectively prior to patient recruitment. Clinical, epidemiology or pathology registries complement the arsenal of clinical studies and may guarantee complete recruitment, valid diagnosis and thus comparability [8]. Their most imminent contribution may be that they could allow recruitment of patients with very rare diseases, in which the knowledge about optimal diagnostic assessment and therapy may be limited, or where insufficient information is available to generate scientific hypotheses based on known estimates of case numbers necessary to answer these questions. Furthermore, epidemiology and pathology registries may be indispensible for a complete epidemiological survey of childhood cancers and for maintaining a high standard in histopathologic diagnosis and classification. According to a strict exegesis, registries are not considered a clinical trial, because these registries do not obligatorily require a prospective definition of precise study questions. Nevertheless, they may fulfill some aspects of clinical studies such as diagnostic studies. In this comment, the different aspects, strengths and weaknesses of clinical studies and registries established in pediatric oncology will be critically evaluated.

Clinical interventional trials explore the clinical efficacy and safety of a certain treatment, device or medical strategy. These include pharmacological and early clinical studies (phases 0 , I), in which first the feasibility of treatment is evaluated. The therapeutic efficacy is tested in open or randomized controlled clinical trials (phases II, III). RCTs and the meta-analysis of sev- eral such trials provide the strongest evidence concerning a specific treatment. Among other features, clinical interventional trials are characterized by a prospective definition of primary and secondary objectives. In some clinical interventional trials prognostic or quality of life studies are integrated as secondary objectives [19]. Furthermore, they ensure high patient safety by structured reporting of adverse events including serious adverse events, data safety monitoring and stopping rules. The randomization may allow scientific evaluation of the scientific hypothesis irrespective of a population based patient recruitment and independent of patient selection. However, in rare entities, the study cohort may be too small for statistically meaningful randomized comparison, even in a global setting. Moreover, in a heterogeneous entity such as pediatric soft tissue sarcoma or even acute lymphoblastic leukemia, only a sub-cohort may be eligible for such a randomized comparison $[1,3,18]$. In some instances, consecutive generations of therapy optimization trials are compared to each other, and changes in therapeutic management are compared [26]. However, these historical comparisons provide weaker evidence compared to randomized comparison, mainly because of a inherent bias associated with medical trends over time, e.g. better management of therapy-associated complications.

There are several options to ensure that patients ineligible for RCTs may not be lost to prospective registration and a structured generation of good evidence. Here, diagnostic studies and registries may play an important role. Both focus on different aspects. In fact, diagnostic studies constitute the stronger tool for generating evidence than pure clinical registries. Diagnostic studies evaluate the impact of a specific diagnostic test in a therapeutic setting that basically represents the current standard treatment recommendation. According to the concept of Sackett and Haynes [22], phase I diagnostic studies may help to distinguish between patients with a specific disease and normal individuals. Phase II studies evaluate whether a specific test result is more likely associated with a target disorder than other test results, while phase III questions focus on the diagnostic accuracy of a specific test in patients, in whom it is clinically reasonable to suspect a specific diagnosis. Thus, phase III studies are strongest in testing the diagnostic usefulness for clinical application [2]. Last, the prognostic impact of a diagnostic test is evaluated in phase IV diagnostic studies [21]. Ideally, it should also be shown whether patients may benefit from a 
specific diagnostic test compared to patients who have not been tested [22].

Of note, diagnostic studies do not include a comparison of different therapeutic strategies. Instead, patients can be treated according to current standards. Nevertheless, documentation of treatment, toxicity and outcome is often required to answer the diagnostic questions. Thus, diagnostic studies may provide a similar overview on the clinical course to interventional trials, without the administrative workload of clinical interventional trials according to drug legislation. Moreover, the improvement of diagnostic accuracy and risk stratification may allow for a substantial improvement of the clinical management of these patients. In addition, these diagnostic studies may also reveal biological phenomena that may have a significant impact on the understanding of a specific disease [13]. The EWOG-MDS studies are a successful example for such a study concept $[21,25]$

As a third approach to the generation of evidence, registries have to be appraised. In pediatric oncology, different types of registries have successfully been established, and much of our current knowledge has been derived from these data. In Germany, the epidemiological German Childhood Cancer Registry and the pathology Childhood Tumor Registry or Brain Tumor Registry provide a comprehensive survey of the spectrum of childhood cancers. These registries have also been the basis for the implementation of numerous diagnostic studies that focus on pathology or genetic aspects $[15,16]$. Thus, the registries have revealed significant information regarding epidemiological risk factors, tumor classification, risk stratification and prognosis. By the inclusion of long-term follow-up queries, these registries are also helpful in the research on long-term sequelae after childhood cancer treatment [12].

The development of clinical registries has seen a significant change during the last decade. The impulse for this change came from the new legislative regulations for clinical studies [20]. About 20 years ago, most pediatric cancers were treated within prospective multicenter therapy optimization trials. However, a considerable number of studies have now been transformed into clinical registries. In some instances, prospective trials have ended recruitment, but the next trial generation has not yet been initiated. Here, clinical registries have been established to cover the interim phase and to provide quality controlled treatment by providing precise recommendations for diagnostics and treatment. For safety reasons clinical, pathology and follow-up data are documented prospectively and annual reports are provided. In contrast to phase IV trails, these registries do not necessarily serve to evaluate a specific study hypothesis or study question. As the registries offer access to central diagnostic confirmation and consultation, central data management and standardization of treatment, these registries are important for maintaining the high therapeutic standard in pediatric oncology patients not enrolled into interventional or diagnostic studies.

For entities, for which no interventional or diagnostic study is currently possible, registries may also play an important role in allowing for a complete registration of all patients reported with this specific diagnosis. It should be considered that the complete registration of a specific diagnosis per se is not a scientific goal, if no scientific questions are evaluated in a sound approach. In fact, compared to clinical interventional or diagnostic trials, registries may be at a certain risk to suffer from several weaknesses in scientific methodology. Therefore, to allow the best possible scientific evaluation of patients, registries have to be designed as carefully as prospective clinical trials. At the same time, they have to respect the border to interventional trials defined by law. They must not be an easy escape from sound scientific evaluation or legislative burden with respect to issues of patient safety. Therefore, whenever possible, especially during interim phases between two interventional trails registries should be used for investigation of diagnostic questions.

In very rare diseases, in which knowledge is very limited, however, registries may be the only way to generate any knowledge on the diseases. Without central registries, evidence about these orphan diseases would never reach beyond single case reports or small retrospective series. In these instances, registries may not necessarily require adherence to a uniform therapeutic recommendation. Since the study parameters integrated in the database must be defined, the potential heterogeneity in treatment has to be considered in advance [4]. However, in order to maintain a high standard of care and to reduce heterogeneity in clinical management, a recommendation reflecting the current standard should be provided [24]. As the competence for this disease will also be highest at the coordination centers, they should also offer consultation in difficult cases [4,23]. In this context, the integration of reference pathology (and genetic) evaluation may be essential, in particular in rare and heterogeneous diseases, as this may allow a uniform classification which could lead to the introduction of a diagnostic standard in these often heterogeneous diseases [6]. This approach has been taken in some disease-specific registries, e.g. for sex cord stromal tumors, pleuropulmonary blastoma or NUT midline carcinomas $[9,14,24]$. However, these disease specific registries mainly fulfill the criteria for diagnostic studies rather than being pure registries. Last but not least, the registries should also be submitted to ethical evaluation and approval.

In conclusion, there is a hierarchy of the scientific impact of registries, diagnostic and clinical interventional studies. Registries certainly constitute an important motor of scientific progress in very rare diseases, as they allow a complete documentation of even highly heterogeneous disorders and may provide a comprehensive survey of the studied disease. However, on the basis of complete registration, study questions should be defined so that diagnostic and ideally, clinical interventional studies can be developed. The latter should still be the standard of management in the major childhood cancers.

\section{Acknowledgement}

The German Registry of Pediatric Rare Tumors (Seltene Tumorerkrankungen in der Pädiatrie, STEP) is supported by the Deutsche Kinderkrebsstiftung.

Conflict of interest: The authors have no conflict of interest to disclose.

\section{References}

1 Asselin BL, Devidas M, Wang $C$ et al. Effectiveness of high-dose methotrexate in T-cell lymphoblastic leukemia and advanced-stage lymphoblastic lymphoma: a randomized study by the Children's Oncology Group (POG 9404). Blood 2011; 118: 874-883

2 Baumann I, Führer M, Behrendt S et al. Morphological differentiation of severe aplastic anaemia from hypocellular refractory cytopenia of childhood: reproducibility of histopathological diagnostic criteria. Histopathology 2012; 61: 10-17 
3 Biondi A, Schrappe $M$, De Lorenzo $P$ et al. Imatinib after induction for treatment of children and adolescents with Philadelphiachromosome-positive acute lymphoblastic leukaemia (EsPhALL): a randomised, open-label, intergroup study. Lancet Oncol 2012; 13: 936-945

4 Bisogno G, Ferrari A, Bien E et al. Rare Cancers in Children - The EXPeRT Initiative: A Report from the European Cooperative Study Group on Pediatric Rare Tumors. Klin Padiatr 2012; 224: 416-420

5 Dueckers G, Guellac N, Arbogast $M$ et al. Evidence and consensus based treatment guidelines 2010 for juvenile idiopathic arthritis by the German Society of Paediatric Rheumatology. Klin Padiatr 2011; 223: 386-394

6 Ellerkamp V, Warmann SW, Vorwerk P et al. Exocrine pancreatic tumors in childhood in Germany. Pediatr Blood Cancer 2012; 58: 366-371

7 Farmand S, Baumann $U$, von Bernuth $\mathrm{H}$ et al. Interdisciplinary AWMF guideline for the diagnostics of primary immunodeficiency. Klin Padiatr 2011; 223: 378-385

8 Ferrari A, Bisogno G, De Salvo GL et al. The challenge of very rare tumours in childhood: the Italian TREP project. Eur J Cancer 2007; 43: 654-659

9 French CA. NUT midline carcinoma. Cancer Genet Cytogenet 2010; 203: $16-20$

10 Garbe C, Peris K, Hauschild A et al. Diagnosis and treatment of melanoma. European consensus-based interdisciplinary guideline - Update 2012. Eur J Cancer 2012; 48: 2375-2390

11 Gortner L, Moller J, Reiss I. ARDS/ALI in Childhood: treatment guideline. Klin Padiatr 2011; 223: 440-444

12 Grabow D, Spix C, Blettner $M$ et al. Strategy for long-term surveillance at the German Childhood Cancer Registry - an update. Klin Padiatr 2011; 223: 159-164

13 Haemmerling S, Behnisch W, Doerks T et al. A 15q24 microdeletion in transient myeloproliferative disease (TMD) and acute megakaryoblastic leukaemia (AMKL) implicates PML and SUMO3 in the leukaemogenesis of TMD/AMKL. Br J Haematol 2012; 157: 180-187

14 Hill DA, Ivanovich J, Priest JR et al. DICER1 mutations in familial pleuropulmonary blastoma. Science 2009; 325: 965
15 Koch A, Denkhaus D, Albrecht S et al. Childhood hepatoblastomas frequently carry a mutated degradation targeting box of the beta-catenin gene. Cancer Res 1999; 59: 269-273

16 Kordes U, Gesk S, Fruhwald MC et al. Clinical and molecular features in patients with atypical teratoid rhabdoid tumor or malignant rhabdoid tumor. Genes Chromosomes Cancer 2010; 49: 176-181

17 Krudewig J, Baumann $U$, Bernuth von $\mathrm{H}$ et al. Interdisciplinary AWMF guideline for the treatment of primary antibody deficiencies. Klin Padiatr 2012; 224: 404-415

18 Le Deley MC, Rosolen A, Williams DM et al. Vinblastine in children and adolescents with high-risk anaplastic large-cell lymphoma: results of the randomized ALCL99-vinblastine trial. J Clin Oncol 2010; 28: 3987-3993

19 Marina N, Bielack S, Whelan J et al. International collaboration is feasible in trials for rare conditions: the EURAMOS experience. Cancer Treat Res 2009; 152: 339-353

20 Ohmann C. Clinical studies in pediatrics: challenges and actual developments. Klin Padiatr 2008; 220: 221-223

21 Olk-Batz C, Poetsch AR, Nollke $P$ et al. Aberrant DNA methylation characterizes juvenile myelomonocytic leukemia with poor outcome. Blood 2011; 117: 4871-4880

22 Sackett DL, Haynes RB. The architecture of diagnostic research. BM] 2002; 324: 539-541

23 Schneider DT, Brecht IB. Care for rare cancers: improved care requires improved communication. Klin Pädiatr 2010; 222: 124-126

24 Schultz KA, Schneider DT, Pashankar F et al. Management of ovarian and testicular sex cord-stromal tumors in children and adolescents. J Pediatr Hematol Oncol 2012; 34: (Suppl 2): S55-S63

25 Thol F, Kade S, Schlarmann $C$ et al. Frequency and prognostic impact of mutations in SRSF2, U2AF1, and ZRSR2 in patients with myelodysplastic syndromes. Blood 2012; 119: 3578-3584

26 Woessmann W, Seidemann K, Mann G et al. The impact of the methotrexate administration schedule and dose in the treatment of children and adolescents with B-cell neoplasms: a report of the BFM Group Study NHL-BFM95. Blood 2005; 105: 948-958 